\title{
Exogenous IGF-1 improves cognitive function in rats with high-fat diet consumption
}

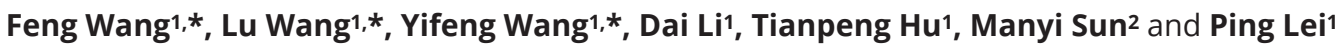 \\ 'Department of Geriatrics, Tianjin Medical University General Hospital, Tianjin Geriatrics Institute, Tianjin, China \\ 2Department of Digestion, Tianjin United Medical Center \& Tianjin People's Hospital, Tianjin, China \\ Correspondence should be addressed to P Lei: leiping_1974@163.com \\ *(F Wang, L Wang and Y Wang contributed equally to this work)
}

\begin{abstract}
Insulin-like growth factor-1 (IGF-1) improves cognitive function, but its mechanism has not been elucidated. The aim of the study was to explore whether IGF-1 exerted its protective effect on cognitive function and anxiety behavior through the activation of PI3K/Akt/ CREB pathway in high-fat diet rats. Neuronal cells HT22 were treated with nothing, IGF1, IGF-1 + LY294002 or IGF-1 + 666-15. Expressions of p-PI3K, p-Akt and p-CREB were measured using Western blot analysis. Thirty $C 57 \mathrm{BL} / 6 \mathrm{~J}$ rats were used. After feeding with high-fat diet, normal saline, PEG-IGF-1, PEG-IGF-1 + LY294002 or PEG-IGF-1 + 666-15 was treated. Cognitive function and anxiety behavior were assessed by Morris water maze and open field test. Several inflammation and oxidative stress biomarkers were measured using recognized methods. Expressions of p-PI3K and p-CREB were also measured using Western blot analysis. After IGF-1 treatment in cells, expressions of p-PI3K, p-Akt and p-CREB were increased. Furthermore, LY294002 downregulated the expressions of these three proteins, but 666-15 only inhibited the expression of CREB in the cells. Compared with the control rats, we found abnormalities of cognitive function and anxiety behavior, inhibition of PI3K/Akt/CREB pathway and increase of oxidative stress and inflammation in high-fat diet rats. After PEG-IGF-1 treatment, the changes in high-fat diet rats were reversed. Then, we blocked the pathway and found that these blockers attenuated the protective effects of PEG-IGF-1. In conclusion, IGF-1 improved cognitive function and anxiety behavior in high-fat diet rats and inhibited inflammation and oxidative stress in hippocampus tissue through the activation of PI3K/Akt/CREB pathway.
\end{abstract}

\section{Introduction}

Cognitive impairment is a common type of condition in the elderly, which includes mild cognitive impairment, Alzheimer's disease, vascular dementia and so on. Over the past decade, more and more evidences have confirmed that metabolic syndrome (MS) contributes to the development of cognitive impairment (Solfrizzi et al. 2010, Watts et al. 2013, Ng et al. 2016). Our studies also report that obesity, hyperlipidemia and hyperglycemia are associated with the increased risk of mild cognitive impairment (Wang et al. 2017a,b,c). Furthermore, it is believed that oxidative stress and inflammation caused by metabolic disorders lead to the quantity reduction and dysfunction of hippocampus neurons, which may partly explain the effect of metabolic disorders on 
cognitive function in elderly (Mukherjee et al. 2018, Zhong et al. 2019).

cAMP-response element-binding protein (CREB) is a transcriptional regulator in the nucleus of neurons, which plays an important role in axonal development, memory formation and neuroprotection (Yu et al. 2017). An in vivo study suggests that high-fat diet causes cognitive decline and down-regulation of CREB in hippocampus (Tang et al. 2018). Our previous study finds that cognitively normal elderly have higher expression of CREB compared with cognitively impaired elderly (Zhang et al. 2019). In addition, CREB can be activated by serine protein kinase (Akt) (Wang et al. 2016). Phosphatidylinositol 3-kinase (PI3K)/Akt-induced CREB activation exhibits neuroprotective effects (Srivastava et al. 2018) and also improves cognitive function in rats (Yang et al. 2018).

Insulin-like growth factor-1 (IGF-1) is a polypeptide which is closely related to the growth and aging of neurons (Frater et al. 2018). IGF-1 binds to its receptor on cell membrane and phosphorylates a variety of intracellular enzymes including PI3K/Akt (Li et al. 2018). A previous study suggests that IGF-1 therapy promotes hippocampus neurogenesis and improves spatial memory in rats (Pardo et al. 2016). High-sugar and high-fat diet decreases the expression of IGF-1 and inhibits the PI3K/Akt pathway in blood vessel tissues (Xi et al. 2010, Mughal et al. 2019).

Taken together, IGF-1 might improve cognitive function caused by high-fat diet through the activation of PI3K/Akt/CREB pathway. In the present study, we tried to verify this hypothesis and further revealed potential antioxidative stress and anti-inflammation effects of IGF-1.

\section{Materials and methods}

The study was approved by the ethics committees of Tianjin Medical University General Hospital.

\section{Cells and experimental design of in vitro test}

Mouse hippocampus neuronal cell line HT22 was purchased from Cell Bank of Chinese Academy of Sciences in China (Lee et al. 2014). The cells were incubated using Dulbecco's modified eagle medium with $10 \%$ fetal bovine serum, 100 unit $/ \mathrm{mL}$ penicillin $\mathrm{G}, 100 \mathrm{mg} / \mathrm{mL}$ streptomycin and $2 \mathrm{mmol} / \mathrm{L}$ L-glutamine at $37^{\circ} \mathrm{C}$ in a $5 \%$ $\mathrm{CO}_{2}$ atmosphere.

The cells were randomly divided into control group (CON group), IGF-1 intervention group (IGF group), PI3K inhibition group (PI3K_I group) and CREB inhibition group (CREB_I group). Each group contained six wells of HT22 cells.

The cells in the IGF group, PI3K_I group and CREB_I group were treated with IGF-1 $(20 \mathrm{ng} / \mathrm{mL})$. Meanwhile, the cells in the PI3K_I group and CREB_I group were separately treated with PI3K blocker LY294002 (5 $\mu \mathrm{mol} / \mathrm{L})$ and CREB blocker 666-15 (10 $\mu \mathrm{mol} / \mathrm{L})$. The cells in the CON group were not treated with any reagents.

Then, viabilities and expression levels of several proteins (i.e. p-PI3K, total-PI3K, p-Akt, total-Akt, p-CREB and total-CREB) in cells were measured.

\section{Animals and experimental design of in vivo test}

Thirty male C57BL/6J rats (4-week-old) were purchased from laboratory animal center, academy of military sciences (Beijing, China). They were housed at room temperature with a $12 \mathrm{~h}$ light: $12 \mathrm{~h}$ darkness cycle for 7 days and were fed with normolipidic diet (D12450B, Research Diets) and adequate water.

The rats were randomly divided into control group (CON group, $n=6)$, MS group ( $n=6$ ), IGF-1 intervention group (IGF group, $n=6$ ), PI3K inhibition group (PI3K_I group, $n=6$ ) and CREB inhibition group (CREB_I group, $n=6)$. Several methods were adopted to determine the sample size in our study. First, the sample size calculated by statistical method was up to 32 in each group. This kind of sample size was too large and previous studies had not adopted such a large sample size, so it was not feasible. Second, we looked up a lot of literature and found that many studies only used five to ten rats in each group. Then, we used this sample size (six rats in each group) to carry out the pre-experiment and found that the statistically positive results can be obtained. Therefore, we finally adopted this sample size.

The rats in the CON group were fed with normolipidic diet (D12450B). The rats in the other four groups were fed with high-fat diet with $60 \%$ of energy from fats, $20 \%$ from carbohydrates and 20\% from proteins for 16 weeks to simulate MS (D12492, Research Diets) (Cifre et al. 2018). Several metabolic indicators (e.g. body mass, blood glucose, HbA1c, total cholesterol and triglyceride) were measured following food restriction for at least $8 \mathrm{~h}$ every 2 weeks. The last measurement was conducted before the first reagent intervention (PEG-IGF-1, LY294002 or 66615), and the results were showed in Supplementary Table 1 (see section on supplementary materials given at the end of this article).

After modeling, the rats in the IGF group, PI3K_I group and CREB_I group were intraperitoneally injected with 
$1 \mathrm{mg} / \mathrm{kg}$ of polyethylene glycol - IGF-1 (PEG-IGF-1) two times a week for 4 weeks. The rats in the PI3K_I group and CREB_I group were separately treated with LY294002 (100 $\mathrm{mg} / \mathrm{kg}$, three times a week) and 666-15 (15 mg/kg, three times a week) for 4 weeks by intraperitoneal injection. The rats in the CON group were treated with normal saline (two times a week) for 4 weeks by intraperitoneal injection.

Then, cognitive functions and anxiety behaviors in rats were assessed. Levels of several oxidative stress and inflammation biomarkers, levels of PEG-IGF-1 and expression levels of several proteins (i.e. p-PI3K, totalPI3K, p-CREB and total-CREB) in hippocampus tissues or cerebrospinal fluids were measured.

\section{Viability test in cells}

MTT assay was adopted $1 \mathrm{~h}$ after these interventions to assess the viability of the HT22 cells according to instructions of a commercial cell proliferation MTT kit (Thermo Fisher Scientific). Optical density (OD) was measured at a wavelength of $590 \mathrm{~nm}$ using a spectrophotometer (Bio-Rad). The OD of control cells were regarded as $100 \%$ viable.

\section{Morris water maze in rats}

After these interventions, Morris water maze (MWM) test was performed to assess learning and memory function of the rats in all the groups by researchers blinded to experimental procedures. Adaptive training was conducted on day 1 . From day 2 to day 5 , the navigation test was performed. The rats were randomly placed in a quadrant of the pool. The escape latency from the beginning to finding the platform was recorded according to an overlooking camera with a video-tracking system (TSE Systems, Bad Homburg, Germany). If the escape latency exceeded $60 \mathrm{~s}$, the rats were guided to the platform for $30 \mathrm{~s}$ and their escape latency was recorded as $60 \mathrm{~s}$. In the following spatial probe test, the platform was removed. Each rat swam for $120 \mathrm{~s}$ in the pool. The percentage of time the rat spent in each quadrant and the number of times it passed the target quadrant was recorded.

\section{Open field test in rats}

Open field test (OFT) was performed to evaluate anxietylike behavior and locomotor activity abnormalities of the rats in all the groups by researchers blinded to experimental procedures. Each rat was located in the center of the field for $5 \mathrm{~min}$, and distance of horizontal motion, number of erection, number of entries in the central area and detention time in the central area were recorded. 'Erection' was defined as two forelimbs leaving the ground and the body being erect.

\section{Cerebral fluid collection and tissue preparation}

Briefly, all rats were killed under anesthesia and their cerebrospinal fluid samples were collected from cisterna magna using a glass capillary tube (Liu \& Duff 2008). Then, their hippocampus tissues were isolated and processed in a homogenization buffer using an electrical homogenizer. The homogenated tissues were centrifuged at $500 \boldsymbol{g}$ for $10 \mathrm{~min}$. Their supernatants were collected and centrifuged again at $15,000 \boldsymbol{g}$ for $10 \mathrm{~min}$. Subsequently, the pellets were suspended in the homogenization buffer mentioned previously for two times and centrifuged at 15,000 $\boldsymbol{g}$ for $5 \mathrm{~min}$. The obtained pellets were finally mixed with homogenization buffer, stored at $3^{\circ} \mathrm{C}$ and adopted for the further oxidative stress and inflammation test.

\section{PEG-IGF-1 measurement in cerebral fluids}

Cerebral fluid levels of PEG-IGF-1 in the MS rats and IGF rats were measured using a commercial enzyme linked immunosorbent assay kit (Abcam), and all experimental processes were carried out according to the manufacturer's instructions.

\section{Oxidative stress test in tissues}

Activity of superoxide dismutase (SOD) was measured using a commercial SOD assay kit according to the manufacturer's protocols (Yang et al. 2019). Levels of reactive oxygen species (ROS) in tissue homogenates were measured using a common and reliable method (Inoue et al. 2019). The fluorescence was measured using a NanoDrop spectrofluorometer (Thermo). Its excitation wavelength was $488 \mathrm{~nm}$ and emission wavelength was $525 \mathrm{~nm}$. Levels of malondialdehyde (MDA) in tissue homogenates were determined through thiobarbituric acid-reactive substances measurement according to kit's instructions (Jiancheng Biochemistry, Nanjing, China) (Yang et al. 2019). Its absorbance was obtained at a wavelength of 532 $\mathrm{nm}$ using the spectrofluorometer (Thermo).

\section{Inflammation test in tissues}

Levels of tumor necrosis factor- $\alpha(\mathrm{TNF}-\alpha)$, interleukin-1 $\beta$ (IL-1 $\beta$ ), interleukin-4 (IL-4) and interleukin-10 (IL-10) 
were measured in fresh tissue homogenates using a commercial enzyme linked immunosorbent assay (ELISA) kit (Thermo Fisher Scientific).

\section{Protein expression test in cells and tissues}

Protein expressions of p-PI3K, total-PI3K, p-Akt, total-Akt, p-CREB and total-CREB in the HT22 cells were measured using Western blot analysis in all the experimental groups. The prepared cells were lysed using Tris-HCl buffer.

Protein expressions of p-PI3K, total-PI3K, p-CREB and total-CREB in fresh hippocampus tissue homogenates were also measured using Western blot analysis in all the experimental groups. The protein was separated from the hippocampus tissue using RIPA lysis and extraction buffer (Thermo Fisher Scientific).

Then, total protein concentrations were measured using Pierce ${ }^{\mathrm{TM}}$ modified Lowry protein assay kit (Thermo Fisher Scientific). Hundred micrograms of protein from each well were resolved using 12\% sodium dodecyl sulfate-PAGE (SDS-PAGE) and transferred onto a BioRad's nitrocellulose membrane. The membrane was blocked using 5\% skimmed milk for $2 \mathrm{~h}$ and incubated with primary antibody anti-p-PI3K (1:1000 dilution), anti-total-PI3K (1:1000 dilution), anti-p-Akt (1:1000 dilution), anti-total-Akt (1:1000 dilution), anti-p-CREB (1:1000 dilution), anti-total-CREB (1:1000 dilution) or anti-GAPDH (1:1000 dilution) at $4^{\circ} \mathrm{C}$ overnight. Then, the membrane was incubated with a horseradish peroxidase (HRP)-conjugated secondary antibody (1:1000 dilution). All primary and secondary antibodies were obtained from Cell Signaling Co. Chemiluminescence detection was performed to visualize the immunoreactive bands (Immobilon Western, Millipore). Intensities of the bands were analyzed using ImageJ software.

\section{Statistical analysis}

Continuous variable in the study was expressed as mean \pm s.D. The difference between two continuous variables was detected using independent sample $t$ test. The difference between more than two continuous variables was measured using variance analysis with LSD test. All the analyses were performed using SPSS 18.0.

\section{Results}

\section{In vivo and in vitro pre-experiments}

In order to avoid potential bias, the effects of reagent IGF-1, PI3K blocker LY294002 and CREB blocker 666-15 on cell activities were compared in all HT22 cell experimental groups. As shown in Fig. 1 and Supplementary Table 2 , there was no significant difference in cell activities among the CON group, IGF group, PI3K_I group and CREB_I group $(P=0.073)$. Thus, these reagents mentioned previously did not affect HT22 cell activities and may not interfere with the conclusions.

Cerebral fluid levels of PEG-IGF-1 were compared in the MS rats and IGF rats. As shown in Fig. 1 and Supplementary Table 4, levels of PEG-IGF-1 in cerebral fluids were significantly higher in the IGF rats than in the MS rats $(P<0.001)$. Therefore, PEG-IGF-1 injected through abdominal cavity can pass through the blood-brain barrier into the cerebrospinal fluid.

\section{Existence of the PI3K/Akt/CREB signal pathway in HT22 cells}

IGF-1, LY294002 and 666-15 were adopted to regulate the expression of p-PI3K, p-Akt and p-CREB in HT22 cells. Through observing changes in the expression of these proteins, the correlation between these proteins was determined and the effect of IGF-1 on the expression of these proteins was also explored. In Fig. 2 and Supplementary Table 3, IGF-1 treatment in the IGF group
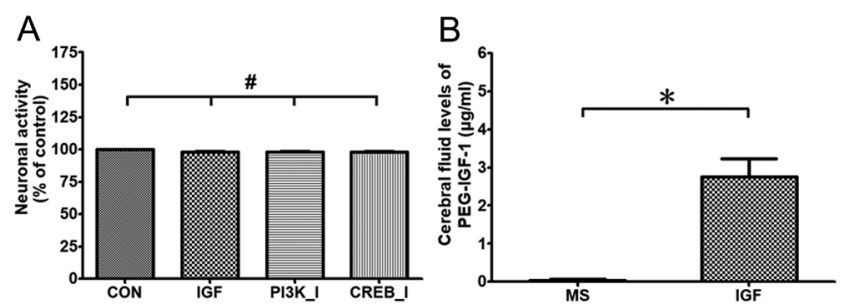

Figure 1

Neuronal activity in all HT22 cell groups and cerebral fluid levels of PEG-IGF-1 in two rat groups. Prepared hippocampal neuronal cell line HT22 were randomly divided into control group (CON group), IGF-1 intervention group (IGF group), PI3K inhibition group (PI3K_I group) and CREB inhibition group (CREB_I group). The cells in the IGF group, PI3K_I group and CREB_I group were treated with IGF-1 (20 ng/mL). Meanwhile, the cells in the PI3K_I group and CREB_I group were separately treated with PI3K blocker LY294002 (5 $\mu \mathrm{mol} / \mathrm{L}$ ) and CREB blocker 666-15 (10 $\mu \mathrm{mol} / \mathrm{L})$. The cells in the CON group were not treated with any reagents. MTT assay was adopted $1 \mathrm{~h}$ after these interventions to assess the cell viability. C57BL/6J rats were randomly divided into metabolic syndrome simulation group (MS group) and IGF-1 intervention group (IGF group). Each group contained six rats. The rats in both groups were fed with high-fat diet. After modeling, the rats in the IGF group were intraperitoneally injected with $1 \mathrm{mg} / \mathrm{kg}$ of polyethylene glycol-IGF-1 (PEG-IGF-1) two times a week for 4 weeks. Cerebral fluid levels of PEG-IGF-1 in the MS rats and IGF rats were measured using ELISA assay. The results are shown as mean \pm S.D. Differences of the results were analyzed using one-way ANOVA with LSD-t test. ' ${ }^{\prime \prime}$ ' indicated $P<0.05$ and '\#' indicated $P>0.05$. 

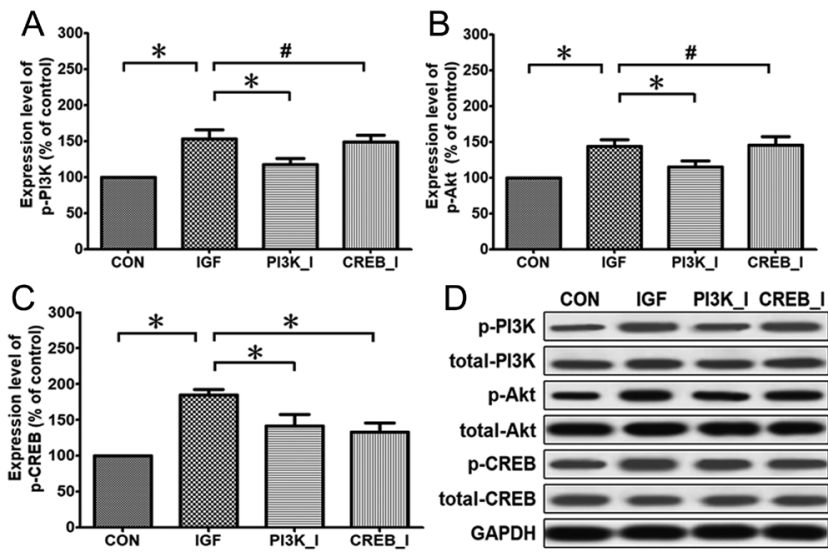

\section{Figure 2}

Protein expressions of $\mathrm{p}-\mathrm{PI} 3 \mathrm{~K}, \mathrm{p}$-Akt and $\mathrm{p}$-CREB in all $\mathrm{HT} 22$ cell groups. Protein expressions of $\mathrm{p}-\mathrm{PI} \mathrm{KK}, \mathrm{p}$-Akt and $\mathrm{p}$-CREB in all the experimental groups were measured using Western blot analysis. The results are shown as mean \pm S.D. Differences of the results were analyzed using one-way ANOVA with LSD-t test. ' ${ }^{*}$ ' indicated $P<0.05$ and ' $\#$ ' indicated $P>0.05$

significantly increased the expressions of p-PI3K, p-Akt and $\mathrm{p}$-CREB proteins compared with the CON group $(P=0.002, P=0.001$ and $P<0.001$, respectively). In the PI3K_I and CREB_I groups, LY294002 downregulated the expressions of all these three proteins $(P=0.046, P=0.39$ and $P=0.037$, respectively), but $666-15$ only inhibited the expression of p-CREB $(P=0.006)$. Therefore, the PI3K/Akt/ CREB signal pathway indeed existed in the HT22 cells, which can be remarkably activated by IGF-1.

\section{IGF-1 improved cognitive function involving the PI3K/Akt/CREB signal pathway in rats}

MWM test was adopted to assess cognitive function (learning and memory function) in rats, and the three markers (i.e. escape latency, percentage of time spent in target quadrant and number of times crossing the platform area) were recorded. In Fig. 3 and Supplementary Table 4 , the escape latencies in MWM test were significantly prolonged in the MS rats than in the CON rats $(P<0.001)$, and the percentage of time spent in target quadrant and number of times crossing the platform area were shorter in the MS rats compared with the CON rats $(P=0.012$ and $P=0.010$, respectively). Furthermore, IGF-1 treatment in the rats significantly attenuated the effects of MS on theses three markers $(P=0.019, P=0.021$ and $P=0.011$, respectively). On the contrary, LY294002 and 666-15 again prolonged the escape latencies (LY294002: $P=0.042$; 666-15: $P=0.045$ ) and reduced the percentage of time spent in target quadrant and number of times crossing
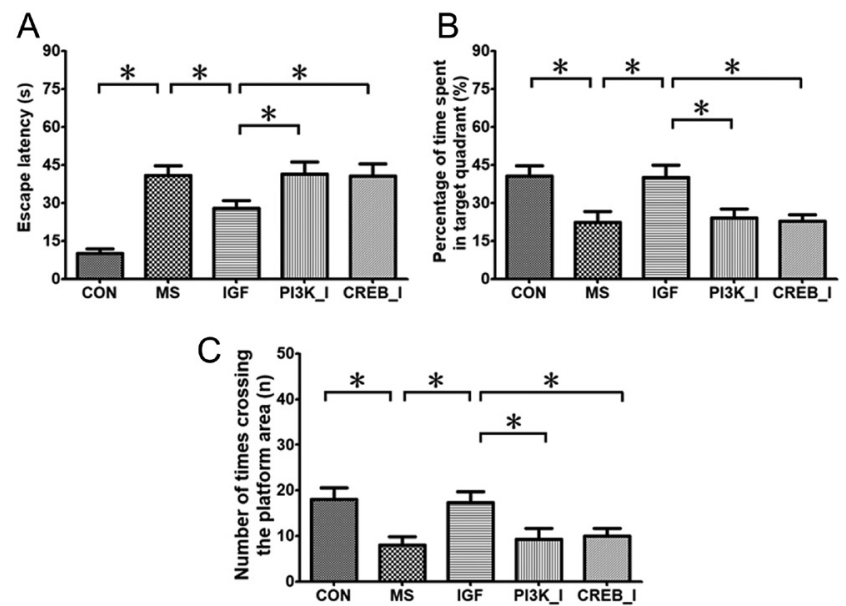

Figure 3

Learning and memory function in all $\mathrm{C} 57 \mathrm{BL} / 6 \mathrm{~J}$ rat groups. C57BL/6J rats were randomly divided into control group (CON group), metabolic syndrome simulation group (MS group), IGF-1 intervention group (IGF group), PI3K inhibition group (PI3K_I group) and CREB inhibition group (CREB_I group). Each group contained six rats. The rats in the MS group, IGF group, PI3K_I group and CREB_I group were fed with high-fat diet. After modeling, the rats in the IGF group, PI3K_I group and CREB_I group were intraperitoneally injected with $1 \mathrm{mg} / \mathrm{kg}$ of polyethylene glycol-IGF-1 (PEG-IGF-1) two times a week for 4 weeks. Meanwhile, the rats in the PI3K_I group were treated with LY294002 (100 mg/kg, three times a week) for 4 weeks by intraperitoneal injection, and the rats in the CREB_I group were treated with 666-15 (15 mg/kg, three times a week) for 4 weeks by intraperitoneal injection. After intervention, Morris water maze test was performed to assess the learning and memory function in all the experimental groups. The results are shown as mean \pm s.D. Differences of the results were analyzed using one-way ANOVA with LSD-t test. '*' indicated $P<0.05$ and '\#' indicated $P>0.05$.

the platform area compared with the rats in the IGF group (LY294002: $P=0.023$ and $P=0.039$, respectively; 66615: $P=0.009$ and $P=0.030$, respectively). These findings indicated that IGF-1 treatment can improve learning and memory impairment caused by MS and that the inhibition of $\mathrm{p}$-PI3K or $\mathrm{p}$-CREB can reverse this protective effect.

\section{IGF-1 improved anxiety like behavior involving the PI3K/Akt/CREB signal pathway in rats}

OFT was adopted to assess anxiety-like behavior and locomotor activity in rats, and the four markers (i.e. distance of horizontal motion, number of erection, number of entries in the central area and detention time in the central area) were recorded. In Fig. 4 and Supplementary Table 4, compared with the CON rats, the distance of horizontal motion and detention time in the central area significantly shortened in the MS rats $(P<0.001$ and $P<0.001$, respectively), and the number of erection and number of entries in the central area remarkably reduced in the MS rats $(P<0.001$ and 
A
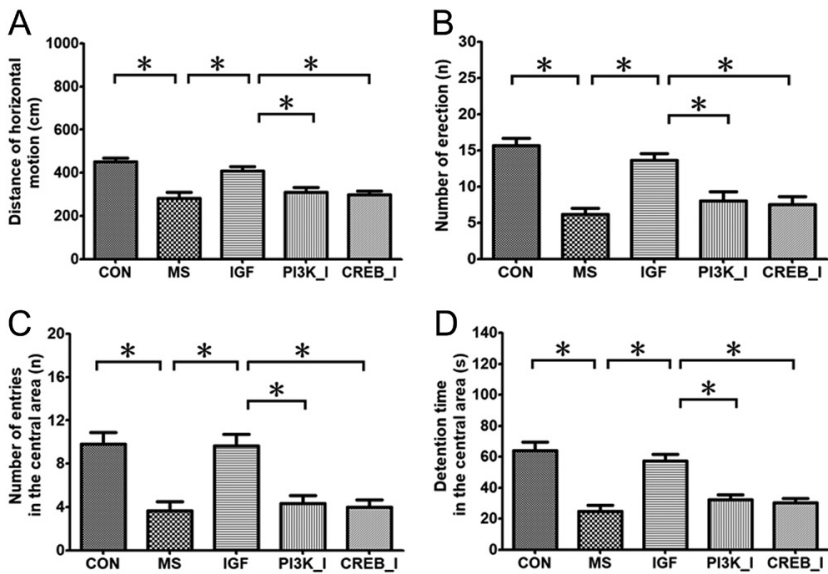

Figure 4

Anxiety-like behavior and locomotor activity in all C57BL/6J rat groups. After intervention, open field test was performed to evaluate anxiety-like behavior and locomotor activity abnormalities of the rats in all the experimental groups. The results are shown as mean \pm S.D. Differences of the results were analyzed using one-way ANOVA with LSD-t test. ‘`' indicated $P<0.05$ and '\#' indicated $P>0.05$.

$P=0.001$, respectively). Similar to the MWM test, IGF-1 treatment can improve the abnormalities of these four markers induced by MS $(P=0.003, P<0.001, P=0.001$ and $P<0.001$, respectively). Furthermore, LY294002 or 666-15 can decrease the distance of horizontal motion, number of erection, number of entries in the central area and detention time in the central area compared with the rats in the IGF group (LY294002: $P=0.006, P=0.005, P=0.002$ and $P=0.001$, respectively; 666-15: $P=0.001, P=0.001$, $P=0.001$ and $P<0.001$, respectively). These findings indicated that IGF-1 treatment can improve anxiety like behavior and locomotor activity impairment caused by MS and that the inhibition of p-PI3K or p-CREB can reverse this protective effect.

\section{Existence of the PI3K/Akt/CREB signal pathway in hippocampus tissues}

IGF-1, LY294002 and 666-15 were adopted to regulate the expression of p-PI3K and p-CREB in hippocampus tissues. And, the existence of the PI3K/Akt/CREB signal pathway and the effect of IGF-1 on these proteins were also explored in rats. In Fig. 5 and Supplementary Table 4, compared with the CON rats, the expressions of these two proteins were down-regulated in the MS rats ( $P=0.002$ and $P<0.001$, respectively). In the IGF rats, IGF-1 treatment again increased the expressions of p-PI3K and $\mathrm{p}$-CREB $(P=0.026$ and $P=0.041$, respectively). In the PI3K_I and CREB_I rats, PI3K blocker LY294002 can inhibit the expression of these two proteins $(P=0.030$

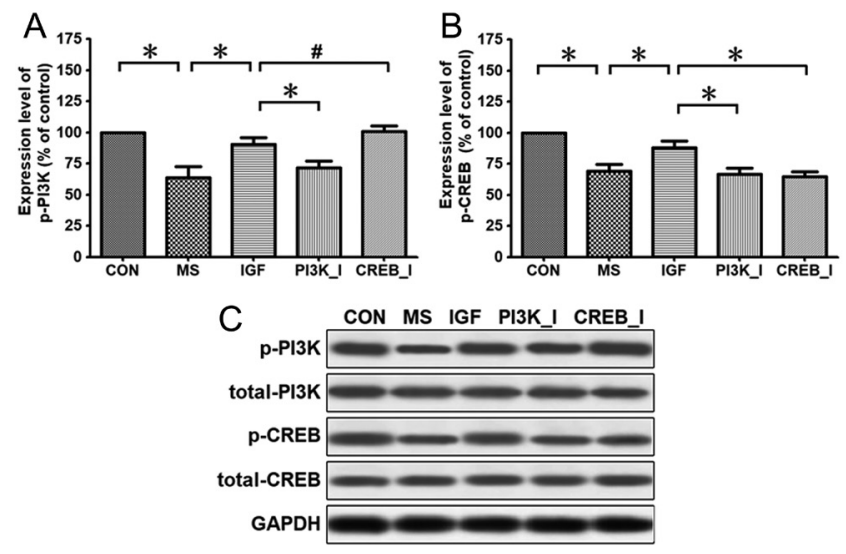

Figure 5

Protein expressions of $\mathrm{p}-\mathrm{PI} 3 \mathrm{~K}$ and $\mathrm{p}$-CREB in all C57BL/6] rat groups. Protein expressions of $\mathrm{p}$-PI3K and $\mathrm{p}$-CREB were measured using Western blot analysis. The results are shown as mean \pm s.D. Differences of the results were analyzed using one-way ANOVA with LSD-t test. ' *' indicated $P<0.05$ and ' $\#$ ' indicated $P>0.05$.

and $P=0.019$, respectively), but CREB blocker $666-15$ can only inhibit the expression of p-CREB $(P=0.009)$. These findings confirmed the existence of the PI3K/Akt/CREB signal pathway in the hippocampus tissues and that the inhibition of the signal pathway caused by MS can be recovered through the treatment of exogenous IGF-1.

\section{IGF-1 improved oxidative stress involving the PI3K/Akt/CREB signal pathway in hippocampus tissues}

Oxidative stress was assessed in hippocampus tissues, and the three biomarkers (i.e. SOD, ROS and MDA) were recorded. In Fig. 6 and Supplementary Table 4, the levels of SOD were decreased in the MS rats compared with the CON rats $(P=0.002)$. After exogenous IGF-1 treatment, the levels of the biomarker were significantly increased $(P=0.026)$. Then, LY294002 and 666-15 can downregulate the levels of SOD again in the PI3K_I rats and the CREB_I rats compared with the IGF rats $(P=0.016$ and $P=0.018$, respectively). On the contrary, the levels of ROS and MDA were increased in the MS rats than in the CON rats ( $P=0.003$ and $P<0.001$, respectively). IGF-1 treatment can inhibit the levels of ROS and MDA in the IGF rats ( $P=0.008$ and $P=0.001$, respectively). Furthermore, the inhibition of p-PI3K and p-CREB elevated the levels of these two biomarkers again (LY294002: $P=0.028$ and $P<0.001$, respectively; 666-15: $P=0.049$ and $P<0.001$, respectively). These findings indicated that IGF-1 relieved oxidative stress caused by MS through activation of the PI3K/Akt/CREB signal pathway in the rats. 

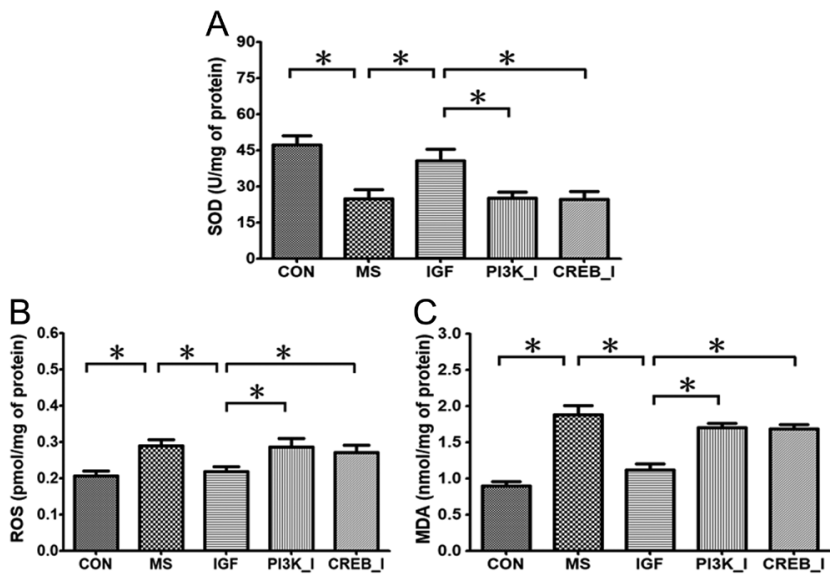

Figure 6

Levels of oxidative stress products and antioxidants in all C57BL/6J rat groups. Levels of superoxide dismutase (SOD), reactive oxygen species (ROS) and malondialdehyde (MDA) were determined using recognized methods. The results are shown as mean \pm S.D. Differences of the results were analyzed using one-way ANOVA with LSD-t test. ' ${ }^{\prime \prime}$ ' indicated $P<0.05$ and ' $\#$ ' indicated $P>0.05$.

\section{IGF-1 improved inflammatory reaction involving the PI3K/Akt/CREB signal pathway in hippocampus tissues}

Inflammatory reaction was assessed in hippocampus tissues, and the four biomarkers (i.e. TNF- $\alpha$, IL-1 $\beta$, IL-4 and IL-10) were recorded. In Fig. 7 and Supplementary Table 4, compared with the CON rats, the MS rats showed the increase of TNF- $\alpha$ and IL- $1 \beta$ levels in the homogenates $(P<0.001$ and $P<0.001$, respectively). Exogenous IGF-1 treatment can decrease the levels of TNF- $\alpha$ and IL-1 $\beta$ $(P<0.001$ and $P<0.001$, respectively). And, the inhibition of $\mathrm{p}$-PI3K and $\mathrm{p}$-CREB have again elevated the levels of these two markers (LY294002: $P=0.002$ and $P<0.001$, respectively; 666-15: $P=0.001$ and $P<0.001$, respectively). Contrarily, the levels of IL-4 and IL-10 were lower in the MS rats than in the CON rats $(P<0.001$ and $P=0.001$, respectively). Compared with the MS rats, IGF-1 treatment has elevated the levels of these two biomarkers $(P=0.004$ and $P=0.002$, respectively). However, the inhibition of p-PI3K and p-CREB again decreased the levels of IL-4 and IL-10 (LY294002: $P=0.027$ and $P=0.003$, respectively; 66615: $P=0.017$ and $P=0.002$, respectively). These findings indicated that IGF-1 relieved inflammatory reaction caused by MS through activation of the PI3K/Akt/CREB signal pathway in the rats.

\section{Discussion}

Before the in vitro study, we determined the activities of the cells which were treated with IGF-1, LY294002 or
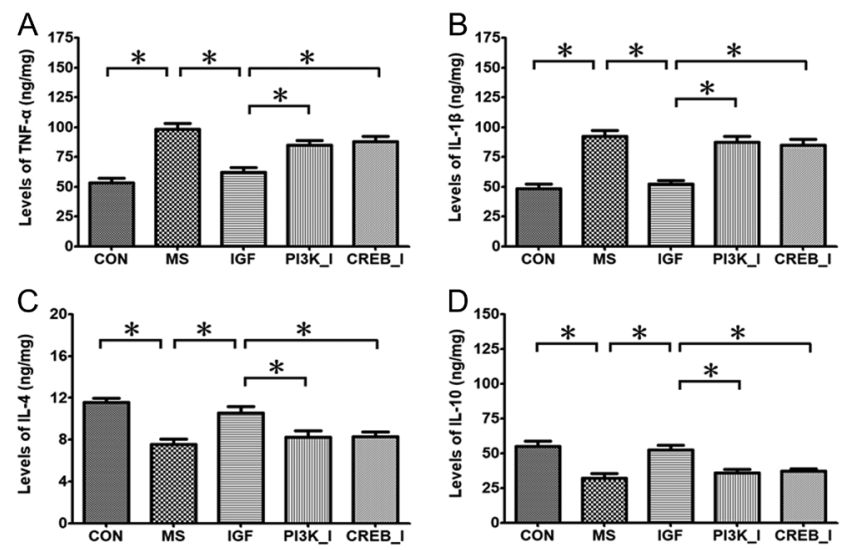

Figure 7

Levels of pro-inflammatory and anti-inflammatory biomarkers in all C57BL/6J rat groups. Levels of tumor necrosis factor- $\alpha$ (TNF- $\alpha$ ), interleukin$1 \beta$ (IL-1 $\beta$ ), interleukin-4 (IL-4) and interleukin-10 (IL-10) were determined using enzyme linked immunosorbent assay. The results are shown as mean \pm S.D. Differences of the results were analyzed using one-way ANOVA with LSD-t test. ' $*$ ' indicated $P<0.05$ and '\#' indicated $P>0.05$.

666-15, and their activities did not undergo a significant change compared with the control cells. So, these reagents in the study may not affect our conclusion.

In the in vitro study, we confirmed that there was a PI3K/Akt/CREB signal pathway in hippocampus neuronal cell line which can be activated by IGF-1. In the in vivo study, we found abnormalities of cognitive function and anxiety behavior in the MS rats, and also found inhibition of the PI3K/Akt/CREB signal pathway in such rats. Exogenous IGF-1 intervention can improve these abnormalities and upregulate the signal pathway in the MS rats. Then, we blocked the pathway separately at two sites ( $\mathrm{p}$-PI3K and $\mathrm{p}$-CREB). We found that the protective effect of exogenous IGF-1 on cognitive function and anxiety behavior was reversed by the inhibition of the signal pathway. Taken together, IGF-1 can improve the cognitive function and anxiety behavior through the activation of the PI3K/Akt/CREB signal pathway in rats with high-fat diet consumption.

In MS, both oxidative stress and inflammation were important factors leading to neuronal damage. Previous studies suggested that CREB can regulate the oxidative stress and inflammation in the nervous system (Motaghinejad et al. 2017, Qi et al. 2017). In the present study, we found that IGF-1 inhibited the oxidative stress and inflammation through the activation of the PI3K/ Akt/CREB signal pathway in the rats, which can partly explain the mechanisms of neuroprotective effects of IGF-1 on MS.

There were several methods to establish an animal model of MS (Ikeda et al. 2006). Gene-knockout model 
ignored the role of the environment in the pathogenesis. Drug induction and surgical operation directly injured the pancreatic $\beta$ cells, which was very different from the pathogenesis of MS. The present study established the model of MS through high-fat diet induction, which was very similar to the occurrence of MS in human. This was more conducive to ensure the reliability of research results.

In the modeling process, the body weights, abdominal circumferences and several biochemical metabolic markers of the rats were regularly tested by one single well-trained researcher. After 16 weeks of modeling, all the rats suffered from abdominal obesity, hyperglycemia, hyperlipidemia and other metabolic disorders, indicating that the rats in the study had been successfully modeled (Supplementary Table 1).

IGF-1, LY294002 and 666-15 were commonly used reagents in vivo studies. Doses of them which referred to previous studies were modified to reduce the number of injections and had also been verified by preliminary experiments (Hu et al. 2000, Xie et al. 2015, Sama et al. 2018). Therefore, there was no ethical problem in the application of these reagents to animals.

MWM test was an experimental method designed by British psychologist Morris in the early 1980s (Mulder $\&$ Pritchett 2003). Nowadays, it had become the most widely recognized method for learning and memory in animal experiments. In the present study, locomotor activity impairment and obese nature in MS rats might confound to the interpretation of the MWM results. However, we did not think these two factors can influence the evaluation of cognitive function. The reasons were listed as follows: First, percentage of time spent in target quadrant (one of the markers in MWM test) was a ratio and was significantly less likely to be affected by locomotor activity impairment. Like other markers in MWM test, this marker produced positive results. Second, IGF-1 treatment significantly improved the results of MWM test, but did not alleviate obesity in MS rats. PI3K blocker LY294002 and CREB blocker 666-15 deteriorated the results of MWM test, but did not promote obesity in MS rats. Therefore, there was no evidence that these two factors can influence the results of MWM test.

There were several limitations and questions for the future in the study. First, IGF-1 was a widely distributed peptide substance and can be produced and secreted by brain tissue itself. The effect of exogenous IGF-1 intervention on the secretion of IGF-1 in brain tissue was not clear. The regulation mechanisms of IGF-1 secretion in brain tissue were also not clear. Second, IGF-1 system included IGF-1, IGF-1 receptor (IGF-1R) and IGF binding proteins (IGFBPs). They collaborated and influenced each other and played a variety of biological roles in the body. However, the effects of IGF-1R and IGFBPs on high-fat dietinduced cognitive dysfunction need further clarification. Third, the downstream signal pathways of IGF-1, which had been revealed, were clearly incomplete and required more comprehensive and systematic research.

In conclusion, both in vivo and in vitro studies confirmed the existence of the PI3K/Akt/CREB signal pathway in the hippocampus neuron, and the signal pathway can be activated by IGF-1. Furthermore, in vivo study also revealed that IGF-1 improved cognitive function and anxiety behavior in the MS rats and inhibited the inflammation and oxidative stress in the hippocampus tissue through the activation of the PI3K/Akt/CREB signal pathway.

\section{Supplementary materials}

This is linked to the online version of the paper at https://doi.org/10.1530/ JME-19-0150.

\section{Declaration of interest}

The authors declare that there is no conflict of interest that could be perceived as prejudicing the impartiality of the research reported.

\section{Funding}

This research did not receive any specific grant from any funding agency in the public, commercial or not-for-profit sector.

\section{References}

Cifre M, Palou A \& Oliver P 2018 Cognitive impairment in metabolically-obese, normal-weight rats: identification of early biomarkers in peripheral blood mononuclear cells. Molecular Neurodegeneration 13 14. (https://doi.org/10.1186/s13024-018-0246-8) Frater J, Lie D, Bartlett P \& McGrath JJ 2018 Insulin-like Growth Factor 1 (IGF-1) as a marker of cognitive decline in normal ageing: a review. Ageing Research Reviews 42 14-27. (https://doi.org/10.1016/j. arr.2017.12.002)

Hu L, Zaloudek C, Mills GB, Gray J \& Jaffe RB 2000 In vivo and in vitro ovarian carcinoma growth inhibition by a phosphatidylinositol 3-kinase inhibitor (LY294002). Clinical Cancer Research 6 880-886.

Ikeda K, Yasui N, Yamamoto J, Nara Y \& Yamori Y 2006 A rat model of metabolic syndrome. Nihon Rinsho 64 339-342.

Inoue T, Hashimoto M, Katakura M, Hossain S, Matsuzaki K \& Shido O 2019 Effect of chronic administration of arachidonic acid on the performance of learning and memory in aged rats. Food and Nutrition Research 63. (https://doi.org/10.29219/fnr.v63.1441)

Lee DS, Ko W, Kim DC, Kim YC \& Jeong GS 2014 Cudarflavone B provides neuroprotection against glutamate-induced mouse hippocampal HT22 cell damage through the Nrf2 and PI3K/Akt signaling pathways. Molecules 19 10818-10831. (https://doi. org/10.3390/molecules190810818)
(C) 2020 Society for Endocrinology Published by Bioscientifica Ltd. Printed in Great Britain 
Li X, Liu H, Wang J, Qin J, Bai Z, Chi B, Yan W \& Chen X 2018 Curcumol induces cell cycle arrest and apoptosis by inhibiting IGF-1R/PI3K/Akt signaling pathway in human nasopharyngeal carcinoma CNE-2 cells. Phytotherapy Research 32 2214-2225. (https:// doi.org/10.1002/ptr.6158)

Liu L \& Duff K 2008 A technique for serial collection of cerebrospinal fluid from the cisterna magna in mouse. Journal of Visualized Experiments 21 e960. (https://doi.org/10.3791/960)

Motaghinejad M, Motevalian M, Babalouei F, Abdollahi M, Heidari M \& Madjd Z 2017 Possible involvement of CREB/BDNF signaling pathway in neuroprotective effects of topiramate against methylphenidate induced apoptosis, oxidative stress and inflammation in isolated hippocampus of rats: molecular, biochemical and histological evidences. Brain Research Bulletin 132 82-98. (https://doi.org/10.1016/j.brainresbull.2017.05.011)

Mughal RS, Bridge K, Buza I, Slaaby R, Worm J, Klitgaard-Povlsen G, Hvid H, Schiødt M, Cubbon R, Yuldasheva N, et al. 2019 Effects of obesity on insulin: insulin-like growth factor 1 hybrid receptor expression and Akt phosphorylation in conduit and resistance arteries. Diabetes and Vascular Disease Research 16 160-170. (https:// doi.org/10.1177/1479164118802550)

Mukherjee A, Mehta BK, Sen KK \& Banerjee S 2018 Metabolic syndromeassociated cognitive decline in mice: role of minocycline. Indian Journal of Pharmacology 50 61-68. (https://doi.org/10.4103/ijp.IJP_110_18)

Mulder GB \& Pritchett K 2003 The Morris water maze. Contemporary Topics in Laboratory Animal Science 42 49-50.

Ng TP, Feng L, Nyunt MS, Feng L, Gao Q, Lim ML, Collinson SL, Chong MS, Lim WS, Lee TS, et al. 2016 Metabolic syndrome and the risk of mild cognitive impairment and progression to dementia: follow-up of the Singapore Longitudinal Ageing Study Cohort. JAMA Neurology 73 456-463. (https://doi.org/10.1001/ jamaneurol.2015.4899)

Pardo J, Uriarte M, Cónsole GM, Reggiani PC, Outeiro TF, Morel GR \& Goya RG 2016 Insulin-like growth factor-I gene therapy increases hippocampal neurogenesis, astrocyte branching and improves spatial memory in female aging rats. European Journal of Neuroscience $\mathbf{4 4}$ 2120-2128. (https://doi.org/10.1111/ejn.13278)

Qi G, Mi Y, Wang Y, Li R, Huang S, Li X \& Liu X 2017 Neuroprotective action of tea polyphenols on oxidative stress-induced apoptosis through the activation of the TrkB/CREB/BDNF pathway and Keap1/ Nrf2 signaling pathway in SH-SY5Y cells and mice brain. Food and Function 8 4421-4432. (https://doi.org/10.1039/C7FO00991G)

Sama DM, Carlson SW, Joseph B, Saenger S, Metzger F \& Saatman KE 2018 Assessment of systemic administration of PEGylated IGF-1 in a mouse model of traumatic brain injury. Restorative Neurology and Neuroscience 36 559-569. (https://doi.org/10.3233/RNN-180831)

Solfrizzi V, Scafato E, Capurso C, D'Introno A, Colacicco AM, Frisardi V, Vendemiale G, Baldereschi M, Crepaldi G, Di Carlo A, et al. 2010 Metabolic syndrome and the risk of vascular dementia: the Italian Longitudinal Study on Ageing. Journal of Neurology, Neurosurgery, and Psychiatry 81 433-440. (https://doi.org/10.1136/ jnnp.2009.181743)

Srivastava P, Dhuriya YK, Kumar V, Srivastava A, Gupta R, Shukla RK, Yadav RS, Dwivedi HN, Pant AB \& Khanna VK 2018 PI3K/Akt/GSK3 $\beta$ induced CREB activation ameliorates arsenic mediated alterations in NMDA receptors and associated signaling in rat hippocampus: neuroprotective role of curcumin. Neurotoxicology 67 190-205. (https://doi.org/10.1016/j.neuro.2018.04.018)

Tang SS, Ren Y, Ren XQ, Cao JR, Hong H, Ji H \& Hu QH 2018 ER and/or ER $\beta$ activation ameliorates cognitive impairment, neurogenesis and apoptosis in type 2 diabetes mellitus mice. Experimental Neurology 311 33-43. (https://doi.org/10.1016/j expneurol.2018.09.002)

Wang Y, Zhang J, Han M, Liu B, Gao Y, Ma P, Zhang S, Zheng Q \& Song X 2016 SMND-309 promotes neuron survival through the activation of the PI3K/Akt/CREB-signalling pathway. Pharmaceutical Biology 54 1982-1990. (https://doi.org/10.3109/13880209.2015.1137 951)

Wang F, Zhao M, Han Z, Li D, Zhang S, Zhang Y, Kong X, Zhang Q \& Lei P $2017 a$ Long-term subclinical hyperglycemia and hypoglycemia as independent risk factors for mild cognitive impairment in elderly people. Tohoku Journal of Experimental Medicine 242 121-128. (https://doi.org/10.1620/tjem.242.121)

Wang F, Zhao M, Han Z, Li D, Zhang S, Zhang Y, Kong X, Sun N, Zhang Q \& Lei P 2017b Hyperuricemia as a protective factor for mild cognitive impairment in non-obese elderly. Tohoku Journal of Experimental Medicine 242 37-42. (https://doi.org/10.1620/ tjem.242.37)

Wang F, Zhao M, Han Z, Li D, Zhang S, Zhang Y, Kong X, Sun N, Zhang Q $\&$ Lei P 2017c Association of body mass index with amnestic and nonamnestic mild cognitive impairment risk in elderly. BMC Psychiatry 17 334. (https://doi.org/10.1186/s12888-017-1493-x)

Watts AS, Loskutova N, Burns JM \& Johnson DK 2013 Metabolic syndrome and cognitive decline in early Alzheimer's disease and healthy older adults. Journal of Alzheimer's Disease 35 253-265. (https://doi.org/10.3233/JAD-121168)

Xi G, Shen X, Radhakrishnan Y, Maile L \& Clemmons D 2010 Hyperglycemia-induced p66shc inhibits insulin-like growth factor I-dependent cell survival via impairment of Src kinase-mediated phosphoinositide-3 kinase/AKT activation in vascular smooth muscle cells. Endocrinology 151 3611-3623. (https://doi.org/10.1210/en.20100242)

Xie F, Li BX, Kassenbrock A, Xue C, Wang X, Qian DZ, Sears RC \& Xiao X 2015 Identification of a potent inhibitor of CREB-mediated gene transcription with efficacious in vivo anticancer activity. Journal of Medicinal Chemistry 58 5075-5087. (https://doi.org/10.1021/acs. jmedchem.5b00468)

Yang Y, Fang H, Xu G, Zhen Y, Zhang Y, Tian J, Zhang D, Zhang G \& Xu J 2018 Liraglutide improves cognitive impairment via the AMPK and PI3K/Akt signaling pathways in type 2 diabetic rats. Molecular Medicine Reports 18 2449-2457. (https://doi.org/10.3892/ mmr.2018.9180)

Yang Z, Li H, Tang Y, Liu X, Liao Q, Fan C \& Wang S 2019 CYP1B1 deiciency ameliorates learning and memory deficits caused by high fat diet in mice. American Journal of Translational Research 11 2194-2206.

Yu XW, Oh MM \& Disterhoft JF 2017 CREB, cellular excitability, and cognition: implications for aging. Behavioural Brain Research 322 206-211. (https://doi.org/10.1016/j.bbr.2016.07.042)

Zhang S, Zhao M, Wang F, Liu J, Zheng H \& Lei P 2019 Relationship between normal weight obesity and mild cognitive impairment is reflected in cognitive-related genes in human peripheral blood mononuclear cells. Psychogeriatrics Epub. (https://doi.org/10.1111/ psyg.12452)

Zhong Y, Zhu Y, He T, Li W, Li Q \& Miao Y 2019 Brain-derived neurotrophic factor inhibits hyperglycemia-induced apoptosis and downregulation of synaptic plasticity-related proteins in hippocampal neurons via the PI3K/Akt pathway. International Journal of Molecular Medicine 43 294-304. (https://doi.org/10.3892/ ijmm.2018.3933)

Received in final form 5 December 2019

Accepted 19 December 2019

Accepted Manuscript published online 19 December 2019
(C) 2020 Society for Endocrinology Published by Bioscientifica Ltd. Printed in Great Britain 